\title{
Las diferencias individuales en sensibilidad a la recompensa modulan la actividad cerebral en una tarea de control inhibitorio con contingencias de reforzamiento
}

\author{
PATRICIA ROSELL-NEGRE \\ prosell@uji.es \\ JuAn CARLos Bustamante \\ jbustama@unizar.es \\ VÍCTOR COSTUMERO \\ vcostume@uji.es \\ PAOLA Fuentes-ClaRAMONTE \\ pfuentes@uji.es \\ NoElia VENTURA-CAMPOS \\ venturan@uji.es \\ Alfonso BARROS-LOSCERTALES \\ barros@uji.es \\ CÉSAR ÁVILA-RIVERA \\ avila@uji.es
}

\section{Resumen}

Introducción: La motivación apetitiva influye sobre procesos cognitivos como el control inhibitorio, y dicha influencia parece asociarse con cambios en la actividad del sistema dopaminérgico. Por otra parte, las diferencias individuales en Sensibilidad a la Recompensa (SR) parecen modular la actividad de las áreas cerebrales que constituyen dicho sistema. El objetivo de este estudio es determinar si las diferencias individuales en SR modulan la actividad cerebral de áreas implicadas en una tarea de control inhibitorio, cuando se recompensa la ejecución correcta a lo largo de la misma. Metodología: Nueve sujetos diestros participaron en el estudio. Se utilizó una tarea de señal de stop con contingencias de reforzamiento monetario. Para medir la SR se empleó el cuestionario SPSRQ de Torrubia y cols. (2001). El procesamiento de las imágenes y el análisis estadístico se realizó mediante el SPM8. Resultados: Los resultados funcionales mostraron un efecto de inhibición ( $p<0.005$, a nivel de vóxel no corregido) en áreas frontales, parietales y estriatales involucradas en procesos de control inhibitorio. Por otra parte, se observó un efecto de interacción inhibición-reforzamiento $(p<0.005$, a nivel de vóxel no corregido) en el giro frontal inferior (GFI) izquierdo y el estriado. Además, se obtuvo una correlación positiva $(r=0.842, p<0.01)$ entre la puntuación en la escala SR y la actividad del GFI. Conclusión: La SR incrementa la actividad del GFI izquierdo du- 
rante la inhibición conductual recompensada monetariamente. Las diferencias individuales en personalidad modulan la interacción entre procesos cognitivos y motivacionales.

Palabras clave: recompensa, control inhibitorio, sensibilidad a la recompensa, personalidad.

\section{Abstract}

Introduction: Appetitive motivation has an effect on cognitive processes such inhibitory control. This effect is suggested to be associated with functional changes in the dopaminergic system. On the other hand, individual differences in Sensitivity to Reward (SR) seem to modulate brain activity in this system. The aim of this study was to find out whether individual differences in SR would modulate brain activity of regions implicated in an inhibitory control task when correct performance was rewarded. Methods: Nine right-handed participants took part in the study. They performed a stop-signal task with monetary contingencies. The SPSRQ questionnaire (Torrubia et al., 2001) was used to measure SR. Image processing and statistical analyses were carried out using SPM8. Results: Our findings showed that the frontal cortex, parietal cortex and striatum, were active during the stop-signal task performance ( $p<0,005$, vóxel-wise uncorrected level). Moreover, a reward-inhibition interaction effect ( $p<0,005$, vóxel-wise uncorrected level) was observed in the left inferior frontal gyrus (IFG) and striatum. Finally, a positive correlation between SR scores and IFG activity $(r=0.842, p<0,01)$ was obtained. Conclusions: SR increases brain activation in the left IFG during behavioral inhibition under reward contingencies. Therefore, individual differences in personality modulate brain activation during the interaction of cognitive and motivational processes.

Keywords: reward, response inhibition, sensitivity to reward, personality.

\section{Introducción}

El comportamiento adaptativo requiere de una constante evaluación de las acciones llevadas a cabo (Pessoa y Engelmann, 2010) y de sus consecuencias, así pues, se puede afirmar que el comportamiento está afectado por factores motivacionales ligados al refuerzo y al castigo (Pessoa y Engelmann, 2010). Padmala y Pessoa (2011) sugieren diferentes formas de modulación motivacional no excluyentes entre si; (1) el aumento de motivación "energiza» la función cognitiva, lo cual hace más veloz la ejecución, (2) la motivación mejora las funciones ejecutivas, y (3) la motivación influye en la selección de la información necesaria para realizar una determinada tarea correctamente. Diversos estudios investigan cómo la motivación puede llegar a afectar a la cognición, y en consecuencia a la conducta, utilizando para ello el refuerzo de la correcta ejecución en diferentes tipos de paradigmas relacionados con funciones de control cognitivo, como la atención (Krebs y cols., 2009; Padmala y Pessoa, 2011; Stoppel y cols., 2011), el control inhibitorio (Padmala y Pessoa, 2010), el cambio de norma (Savine y Braver, 2010), la memoria de trabajo (Beck y cols., 2010; Gilbert y Fiez, 2004) o la toma de decisiones (Pochon y cols., 2002; Rogers y cols., 2004). Un ejemplo de ello es el estudio de Tomporowski y Tinsley (1996), en el que utilizando una tarea de atención sostenida, observaron que cuando los participantes eran reforzados económicamente por su participación conseguían 
mantener la atención, mientras que cuando no se les recompensaba la atención sostenida disminuía significativamente.

Los resultados funcionales obtenidos han mostrado que la red neuronal involucrada en la interacción entre el control cognitivo y la motivación apetitiva incluye áreas (1) frontales como el córtex prefrontal medio o el giro frontal inferior, (2) parietales como el sulco intraparietal o ínsula, y (3) estriatales como el nucleo accumbens, o el putamen (Padmala y Pessoa, 2011; Stoppel y cols., 2011; Padmala y Pessoa, 2010; Savine y Braver, 2010; Beck y cols., 2010; Pochon y cols., 2002; Rogers y cols., 2004). De igual modo, los apuntan a la dopamina como principal neurotransmisor involucrado en esta interacción (Engelmann y cols., 2009; Zink y cols., 2004; Pessoa y Engelmann, 2010; Ávila y cols., 2012). Según Aarts y cols. (2011) la dopamina dirige la información desde los circuitos frontoestriatales más ventromediales (p. ej.: núcleo accumbens y córtex orbitofrontal) implicados en la recompensa y la motivación apetitiva, hasta circuitos frontoestriatales (p. ej.: caudado, putamen y córtex prefrontal dorsolateral) dorsolaterales asociados con la cognición y la acción.

La influencia sobre la cognición y la conducta por parte de la motivación apetitiva, vendría definida por las diferencias individuales en rasgos de personalidad (Locke y Braver, 2008) como por ejemplo, la Sensibilidad a la Recompensa (SR). La SR es un rasgo de personalidad relacionado con el Sistema de Aproximación Conductual (SAC), el cual define las diferencias individuales en la sensibilidad y reactividad a los estímulos apetitivos, y se relaciona con la actividad de estructuras mesocorticolímbicas (Ávila y cols., 2008). Al igual que sucedía con la interacción cognitivo-motivacional Ávila y cols., (2012) apuntan a la dopamina como principal neurotransmisor implicado en la regulación de la actividad del SAC. Estudios como los de Locke y Braver (2008) y Simon y cols. (2010) investigaron a nivel neuronal la interacción entre la atención y la motivación apetitiva utilizando tareas de atención con contingencias de refuerzo, y analizaron la influencia de la activación del SAC sobre esta interacción. En el primero de los estudios mencionados, observaron que aquellos participantes con un alto SAC mostraban mayor activación del estriado ventral cuando recibían la recompensa por la correcta ejecución. Mientras que Locke y Braver (2008), hallaron que áreas como el córtex orbitofrontal (COF), caudado y córtex prefrontal (CPF), correlacionaban positivamente con altas puntuaciones en el SAC. En la misma línea, Ávila y cols., (2012), examinaron la asociación entre las puntuaciones en SR y las áreas cerebrales involucradas en una tarea de cambio de set, observando que la actividad del giro frontal inferior (GFI) y el estriado, áreas relacionadas con la ejecución de dicha tarea, estaba a su vez modulada por las diferencias individuales en SR.

El presente estudio se centrará en un proceso de control cognitivo como es la inhibición de respuesta. La inhibición de respuesta es la habilidad o capacidad de suprimir una acción en respuesta a cambios en el estado interno o en el entorno de un individuo, de donde subyacen un amplio rango de comportamientos críticos en el funcionamiento adaptativo (Congdon y cols., 2012). Este tipo de procesos suelen ser estudiados con tareas «go/no-go» o tareas de señal de stop (del inglés, stop-signal task). Diversos estudios han mostrado la implicación de áreas como el córtex frontal inferior derecho (CFI derecho), tálamo bilateral, córtex frontal medial (área premotora y córtex cingulado anterior), estriado, córtex parietal (CP) y córtex orbitofrontal (COF) bilateral en este tipo de tareas de inhibición conductual (Aron y Poldrack, 2006; Aron y cols., 2007; Verbruggen y Logan, 2008). El objetivo de nuestro estudio es analizar: (1) si la motivación apetitiva influye sobre la actividad cerebral durante la ejecución de una tarea de señal de stop, y (2) si tal influencia se ve modulada por diferencias individuales en el rasgo de personalidad de SR. Se predice que: (1) Existirán diferencias en la actividad de las áreas cerebrales implicadas en la tarea de señal de stop, cuando haya contingencia de reforzamiento en comparación a cuando no las haya; y que (2) las diferencias individuales en SR correlacionarán de forma positiva con la actividad de las áreas cerebrales implicadas en procesos cognitivos de inhibición cuando la correcta ejecución sea recompensada. 


\section{Métodos}

\section{Participantes}

En este estudio participaron 9 voluntarios diestros ( 8 hombres y 1 mujer) con una media de edad de 36,44 años (D. S. = 7,57; rango $=27-47$ años) y una media de años de educación de 13,44 años (D. S. $=3,5$; rango = 8-17 años). Los criterios de inclusión bajo los que se realizó la selección de la muestra fueron: (1) no tener enfermedad médica o neurológica importante o trastorno psicopatológico, (2) no tener antecedentes de traumatismo craneoencefálico con pérdida de conciencia superior a 30 minutos, y (3) sin consumo actual de medicamentos o sustancias psicoactivas.

Todos los participantes completaron el Cuestionario de Sensibilidad al Castigo y Sensibilidad a la Recompensa (SCRSQ; Torrubia, Ávila, Moltó y Caseras, 2000), obteniendo una puntuación media para la SR de 12,67 (D. S. = 3,43; rango $=8-18$ ). Además, fueron informados sobre la naturaleza de la investigación y firmaron un consentimiento informado por escrito. Al finalizar se les pagó por su participación según su ejecución. El estudio fue aprobado por el Comité de Ética de la Universitat Jaume I de Castellón.

\section{Paradigma}

La tarea utilizada fue una tarea de señal de stop con contingencias de refuerzo monetario (véase figura 1). En las tareas de señal de stop los participantes deben responder a un estímulo en función de alguna característica del mismo (ensayos go). Ocasionalmente, este estímulo al que el participante debe responder, va seguido de una señal de stop que indica a los participantes que deben detener su respuesta (ensayos stop). Para introducir el componente motivacional apetitivo en el paradigma, se recompensaron económicamente los ensayos correctamente ejecutados (tanto los ensayos go como los ensayos stop) a lo largo de la tarea.

En los ensayos go se presentaba un cuadrado con un círculo pequeño dentro del mismo (estímulo-respuesta; ER). La tarea de los participantes consistía en indicar si el círculo estaba en la parte superior o inferior dentro de un cuadrado. En las instrucciones, que fueron leídas a cada uno de los participantes, se les pedía que respondieran tan rápido como pudieran durante la presentación del ER, ya que las respuestas que se dieran fuera de este tiempo serían tratadas como erróneas. Los ensayos stop, eran idénticos a los go, con la excepción que tras un intervalo de tiempo variable denominado «retraso de la señal de stop» (SSD, del inglés stop signal delay) se presentaba un cuadrado tachado con un aspa (señal-stop; sS) que indicaba a los participantes que debían detener la respuesta que habían iniciado tras la presentación del ER. EI SSD se ajustaba dinámicamente durante toda la tarea dependiendo de la ejecución del participante en el ensayo stop previo dentro de un rango temporal de 50 a $850 \mathrm{~ms}$. Si el participante conseguía inhibir, el SSD aumentaba 50 ms haciendo la inhibición más difícil, mientras que si el participante no conseguía inhibir su respuesta en el ensayo previo el SSD disminuía 50 ms haciendo la tarea más fácil. Este procedimiento, conocido con el nombre de staircasing asegura que los participantes consigan inhibir la respuesta en aproximadamente el $50 \%$ de los ensayos stop (Logan y cols., 1997; Logan y Cowan, 1984).

Tanto los ensayos go como los stop se secuenciaron de forma pseudoaleatoria, comenzando con la presentación de un punto de fijación durante $500 \mathrm{~ms}$ con el objetivo de captar la atención del participante. Tras este punto de fijación y antes del ER, aparecía una figura geométrica durante $1 \mathrm{~s}$ (señal; $\mathrm{s}$ ) que indicaba si ese mismo ensayo iba o no a ser recompensado económicamente por su correcta ejecución. La aparición de un círculo informaba de que la correcta ejecución en ese mismo ensayo iba a ser recompensada económicamente con 0,20 euros (condición-recompensa), mientras que la aparición de un triángulo informaba de que no se les iba a recompensar (condición-control). Tras un intervalo de tiempo variable de 2, 3 ó 4 segundos 
(intervalo entre S y ER; ISI señal) aparecía el ER (durante 1 segundo) al que los participantes debían responder. Tras el ER, los sujetos veían una pantalla en negro durante 1, 2 o 3 segundos (intervalo entre el ER y el feedback; ISI feedback). Por último, se presentaba el feedback (F) correspondiente a ese ensayo durante $500 \mathrm{~ms}$, el cual dependía de la condición experimental en la que se encontraba el ensayo (recompensa o control) y de la ejecución del participante. Dentro de los ensayos go, un acierto se consideraría responder adecuadamente a la posición del círculo pequeño establecida de forma pseudoaleatoria dentro del cuadrado, mientras que en los ensayos stop, un acierto sería conseguir inhibir la respuesta. Respecto a la pseudoaleatorización de los ensayos stop, nunca se presentaron dos ensayos stop seguidos, y se intercalaron de 1 a 4 ensayos go por cada ensayo stop de forma pseudoaleatorizada, teniendo en cuenta que el número de ensayos intercalados fuera el mismo para los ensayos de la condición recompensa y condición control. Tras el $\mathrm{F}$ volvía a aparecer una pantalla en negro con una duración variable de entre 1 y 4 segundos (intervalo entre ensayos) siguiendo una distribución exponencial de 1 a 4 segundos con una media lo más próxima posible a 1 segundo.

Todos los sujetos realizaron 2 bloques de 170 ensayos cada uno, lo cual hace un total de 340 ensayos, de los cuales 100 eran ensayos stop (30\%) y el resto go. A su vez, de los 340 ensayos, la mitad pertenecían a la condición control y la otra mitad a la condición recompensa. Por lo que obtuvimos 120 ensayos go-condición control, 120 ensayos go-condición recompensa, 50 ensayos stop-condición control y 50 ensayos stop-condición recompensa. La duración media de cada ensayo fue de 7,30 segundos, con lo cual cada bloque duraba aproximadamente 20,71 minutos. Los bloques estaban separados por un descanso de 2 minutos. En el feedback correspondiente al último ensayo de cada bloque aparecía además el dinero que el participante llevaba acumulado hasta ese momento.

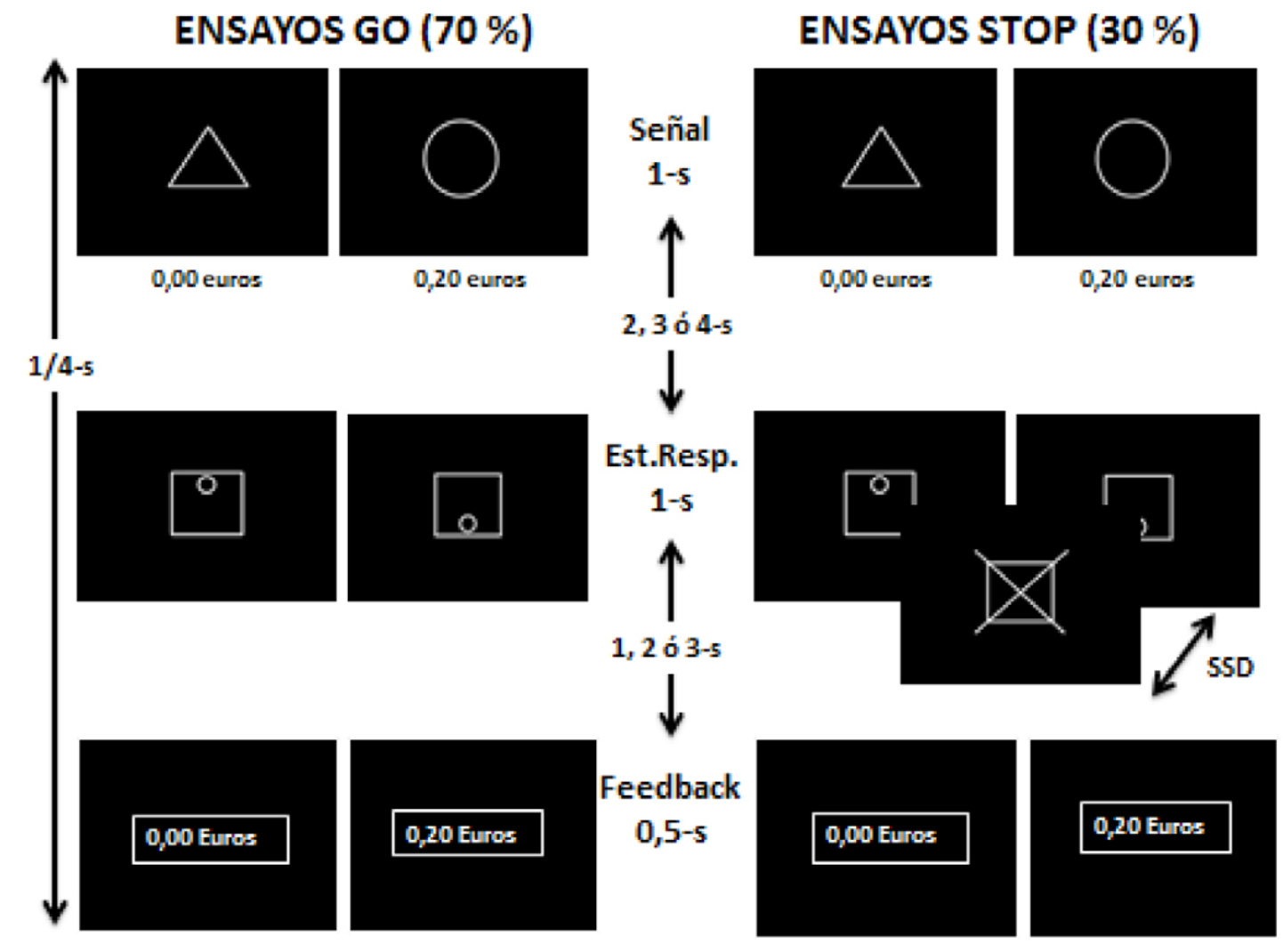

Figura 1. Tarea stop-signal 
Antes de entrar en el escáner, todos los participantes recibieron las mismas instrucciones de la tarea y se les informó de que recibirían una recompensa económica al finalizar su participación que dependería de su ejecución a lo largo de la tarea. Una vez dentro del escáner y durante la adquisición del volumen anatómico, completaron una versión práctica de la tarea de una duración de 11,36 minutos. De esta tarea práctica se extrajo la mediana de los SSD de cada una de las condiciones experimentales (recompensa y control) para cada participante y ambos valores fueron introducidos como SSD iniciales en la tarea dentro del escáner. Como hemos dicho anteriormente, el SSD se ajustaba dinámicamente durante toda la tarea, pero lo hacía de forma independiente para cada una de las condiciones de la tarea, es decir, si el participante no conseguía inhibir su respuesta en un ensayo dentro de la condición control, esto afectaría al SSD del siguiente ensayo control. De igual modo, un error en la inhibición dentro de la condición recompensa solo afectaría al siguiente ensayo dentro de la condición recompensa.

\section{Adquisición de datos de fMRI}

Los datos de RMF basados en el contraste del nivel de sangre oxigenada (BOLD, del inglés Blood Oxigenated Level Dependent) fueron adquiridos en una Siemens Avanto de 1,5 T (Erlangen, Alemania). Los participantes fueron colocados en una posición supina en el escáner de Resonancia Magnética (RM). Sus cabezas fueron inmovilizadas con cojines para reducir los artefactos producidos por el movimiento. El paradigma fue presentado en resonancia utilizando las gafas Visuastim XGA con una resolución de 800 x 600 (Resonance Technologies, Inc., CA, EE. UU.), y con un sistema de dos botones de respuesta (ResponseGrip, NNL, Noruega).

Se utilizó una secuencia gradiente-echo T2* echo-planar RM fue utilizada para la adquisición de las imágenes funcionales $\left(\mathrm{TE}=48 \mathrm{~ms}, \mathrm{TR}=2000 \mathrm{~ms}\right.$, flip angle $=90^{\circ}$, matriz $=64 \times 64$, tamaño de vóxel $=3.5 \times 3.5$, con $4.5 \mathrm{~mm}$ de grosor y $0.5 \mathrm{~mm}$ de separación entre cortes). Se adquirieron 24 cortes axiales intercalados paralelos al plano de la comisura anterior-comisura posterior cubriendo el cerebro entero. Antes de la secuencia de RM (Resonancia Magnética) funcional se adquirió un volumen $3 \mathrm{D}$ anatómico usando una secuencia $\mathrm{T} 1$ gradiente-echo ( $\mathrm{TE}=$ $4.9 \mathrm{~ms}, \mathrm{TR}=11 \mathrm{~ms}, \mathrm{FOV}=224 \mathrm{~mm}$, matriz= $256 \times 256 \times 160$, tamaño de vóxel= $1 \times 1 \times 1)$.

\section{Preprocesado de las imágenes}

El preprocesado de las imágenes y los análisis estadísticos se realizaron con el programa SPM8 (Statistical Parametric Mapping, Wellcome Institute of Cognitive Neurology, Londres $\mathrm{RU})$. Todos los volúmenes funcionales fueron realineados al primer volumen funcional y corregidos por movimiento. Además se corregistraron con el anatómico correspondiente y se normalizaron en base a un espacio estereotáxico estándar (plantilla proporcionada por el Instituto Neurológico de Montreal) con los parámetros obtenidos de la segmentación anatómica. Se aplicó un suavizado espacial con un filtro gaussiano de $8 \mathrm{~mm}$. Además, la serie temporal de la respuesta hemodinámica fue filtrada con un filtro de alta frecuencia (128 s) para eliminar componentes de baja frecuencia. 


\section{Análisis estadístico}

\section{Datos conductuales}

Se utilizó el programa Statistical Package for the Social Sciences (SPSS 20.0 para Windows, SPSS Inc., EE. UU.). El tiempo de reacción para la señal de «stop» (SSRT, del inglés stop-signal reaction time) proporciona una estimación del «tiempo de reacción inhibitorio», y fue calculado restando la media del SSD al tiempo de reacción (TR) medio de los ensayos go correctos (Verbruggen y cols., 2008). El análisis de la ejecución conductual se realizó calculando las medias de las variables conductuales de interés (TR para los ensayos go correctos, SSRT y porcentaje de aciertos de los ensayos stop) por separado para la condición control y condición recompensa (véase tabla 1). Se aplicó una prueba T para muestras relacionadas con el objetivo de averiguar si las diferencias entre ambas condiciones eran estadísticamente significativas.

\section{- Datos fMRI}

El análisis estadístico se realizó sobre los datos individuales y grupales usando el Modelo Lineal General (Friston, 1995). Este método corrige autocorrelaciones temporales y espaciales en los datos de RMF. En el análisis de primer nivel (efectos fijos), las series temporales de cada participante obtenidas del pre-procesado se modelaron para cada condición de interés, utilizando la función de respuesta hemodinámica y su derivada temporal. Los parámetros de corrección de movimiento de cada sujeto se incluyeron en el modelo como regresores de no interés. Se extrajeron nueve contrastes por separado para cada sujeto. Las activaciones o efectos cerebrales con respecto a (1) go correcta condición recompensa, (2) go correcta condición control , (3) stop correcto condición recompensa, (4) stop correcto condición control, (5) stop erróneo condición recompensa (6) stop erróneo condición control. A continuación, las imágenes de contraste obtenidas del análisis de primer nivel se utilizaron en un segundo nivel (efectos aleatorios) para obtener la activación cerebral grupal relacionada con el control inhibitorio y las diferencias entre condiciones de recompensas.

Para calcular el efecto cerebral de inhibir correctamente (efecto de inhibición) y el efecto cerebral de la interacción entre inhibir correcta o incorrectamente y obtener o no una recompensa monetaria (efecto de interacción), se realizó un análisis estadístico vóxel a vóxel a nivel de todo el cerebro utilizando una ANOVA 2 Inhibición (correctas, incorrectas) x 2 Condición (recompensa, control) de medidas repetidas a un nivel $p<0.005$ sin corregir para múliples comparaciones. En el caso del efecto principal de inhibición se realizó un contraste inhibiciones correctas vs. inhibiciones incorrectas incluyendo tanto los ensayos de la condición control como los ensayos de la condición recompensa. Mientras que para estudiar la interacción cognitivo motivacional, se utilizó el mismo contraste, pero teniendo en cuenta si los ensayos pertenecían a la condición control o recompensa.

Siguiendo estudios previos (Hahn y cols., 2009; Gray y cols., 2005; Simon y cols., 2010), el análisis de los efectos de la SR sobre las áreas cerebrales involucradas en la interacción cognitivo-motivacional, se llevó a cabo correlacionando las puntuaciones individuales de nuestros participantes en SR y el valor medio de la actividad en las áreas involucradas de dicha interacción. El análisis se restringió a los volúmenes de interés (Vol, del inglés volumes of interest) en las áreas cerebrales que se observaron implicadas en la interacción cognitivo-motivacional en el presente estudio. Para identificar las diferentes vol se utilizaron las coordenadas del pico máximo de activación. Cada una de las vol consistió en una esfera de $8 \mathrm{~mm}$ de radio centrada en el vóxel que mostró el pico de activación. Para cada uno de los participan- 
tes, se calculó la media de la señal BOLD de los vóxels activos dentro de esa vol. Por último, esos valores fueron correlacionados con las puntuaciones de SR. El umbral del análisis de correlación se estableció en $p<0.05$.

\section{Resultados}

\section{Conductuales}

Los resultados conductuales de interés aparecen resumidos en la tabla 1. Se observaron diferencias significativas $(p<0,05)$ en el TR medio para responder al ER en los ensayos go correctos, siendo menor en los ensayos reforzados que en los controles. Críticamente, se observó una tendencia hacia la significatividad $(p=0,0535)$ en la variable SSRT, siendo menor de nuevo en la condición recompensa que en la control. La correcta ejecución en los ensayos stop fue aproximadamente del $50 \%$ para ambas condiciones.

Tabla 1

Media (desviación estándar) de las variable conductuales de interés en la condición control y en la condición de refuerzo de la tarea

\begin{tabular}{lrrr}
\hline & \multicolumn{1}{c}{ CONTROL } & REFUERZO & SIGNIFICACIÓN $^{*}$ \\
\hline TR ensayos GO $(\mathrm{ms})$ & $645,71(89,79)$ & $621,77(95,76)$ & 0,02 \\
Inhibiciones correctas $(\%)$ & $51.6(7,41)$ & $52(8.86)$ & 0,47 \\
SSD $(\mathrm{ms})$ & $476.29(188.98)$ & $478.54(184.71)$ & 0,3 \\
SSRT $(\mathrm{ms})$ & $167.34(151.32)$ & $145.36(123.12)$ & 0,05 \\
TR ensayos STOP incorrectos $(\mathrm{ms})$ & $602,91(111,37)$ & $602,24(108,86)$ & 0,48 \\
Errores ensayos GO $(\%)$ & $7.6(7.38)$ & $5.45(4.74)$ & 0,09 \\
\hline
\end{tabular}

TR, tiempo de reacción; ms, milisegundos; SSD, stop-signal delay; SSRT; stop-signal reaction time ${ }^{*}$ Diferencias significativas entre condiciones $(p<0,05)$

fMRI

Los efectos de inhibición (véase tabla 2) se observaron en el giro frontal inferior (GFI) izquierdo (figura 2, a), giro frontal superior (GFS) izquierdo (figura 2, b), en el córtex cingulado anterior (CCA) (figura 2, c), giro parietal inferior (GPI) izquierdo (figura 2, d), y estriado derecho (figura 2, e). 
Tabla 2

Efectos cerebrales para los análisis de interés

\begin{tabular}{lccccccc}
\hline $\begin{array}{c}\text { ANÁLISIS } \\
\begin{array}{c}\text { LOCALIZACIÓN (REGIÓN } \\
\text { CEREBRAL) }\end{array}\end{array}$ & $\begin{array}{c}\text { ÁREA DE } \\
\text { BROADMAN }\end{array}$ & LATERALIZACIÓN & $\mathrm{X}$ & $\mathrm{Y}$ & $\mathrm{Z}$ & $\mathrm{K}$ & $\mathrm{T}$ \\
\hline $\begin{array}{l}\text { EFECTO DE INHIBICIÓN } \\
\text { Giro frontal inferior }\end{array}$ & 10 & Izq. & -27 & 53 & -2 & 162 & 3,60 \\
Giro frontal superior & 8 & Izq. & -39 & 29 & 46 & 190 & 4,79 \\
Córtex cingulado anterior & 32 & Izq. & -3 & 41 & 1 & 113 & 4,91 \\
Giro parietal inferior & 39 & Izq. & -39 & -55 & 25 & 963 & 4,69 \\
Estriado & & Der. & 12 & 11 & -5 & 128 & 3,91 \\
& & & & & & & \\
EFECTO DE INTERACCIÓN & 10 & Izq. & -42 & 35 & 4 & 14 & 3,31 \\
Giro frontal inferior & & Der. & 15 & 5 & -5 & 18 & 3,66 \\
Estriado & & & & & & & \\
\hline
\end{tabular}

Izq., izquierdo; der., derecho; k, extensión del cluster de activación obtenido; t, puntuación estadística obtenida para dicho efecto; $\mathrm{x} / \mathrm{y} / \mathrm{z}$, coordenadas MNI en los tres ejes

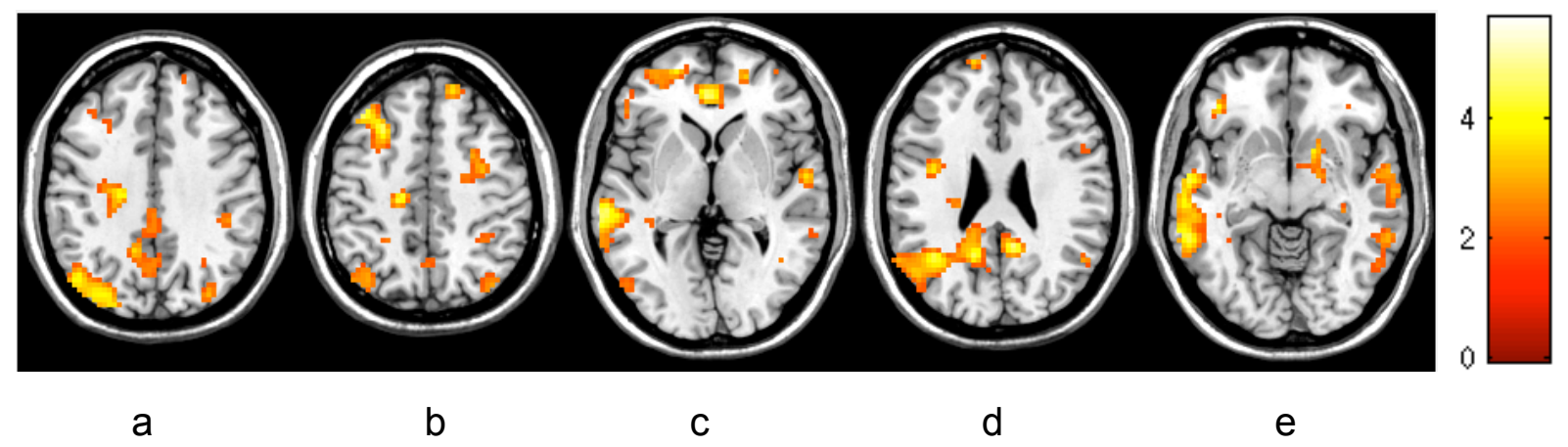

Figura 2. Efectos de inhibición ( $p<0,005$, a nivel de vóxel no corregido)

Se obtuvo un efecto de interacción condición por inhibición en el GFI izq. (figura 3, a) y en el estriado derecho (figura 3, b).

La barra de colores representa los valores estadísticos del efecto de inhibición. 
a
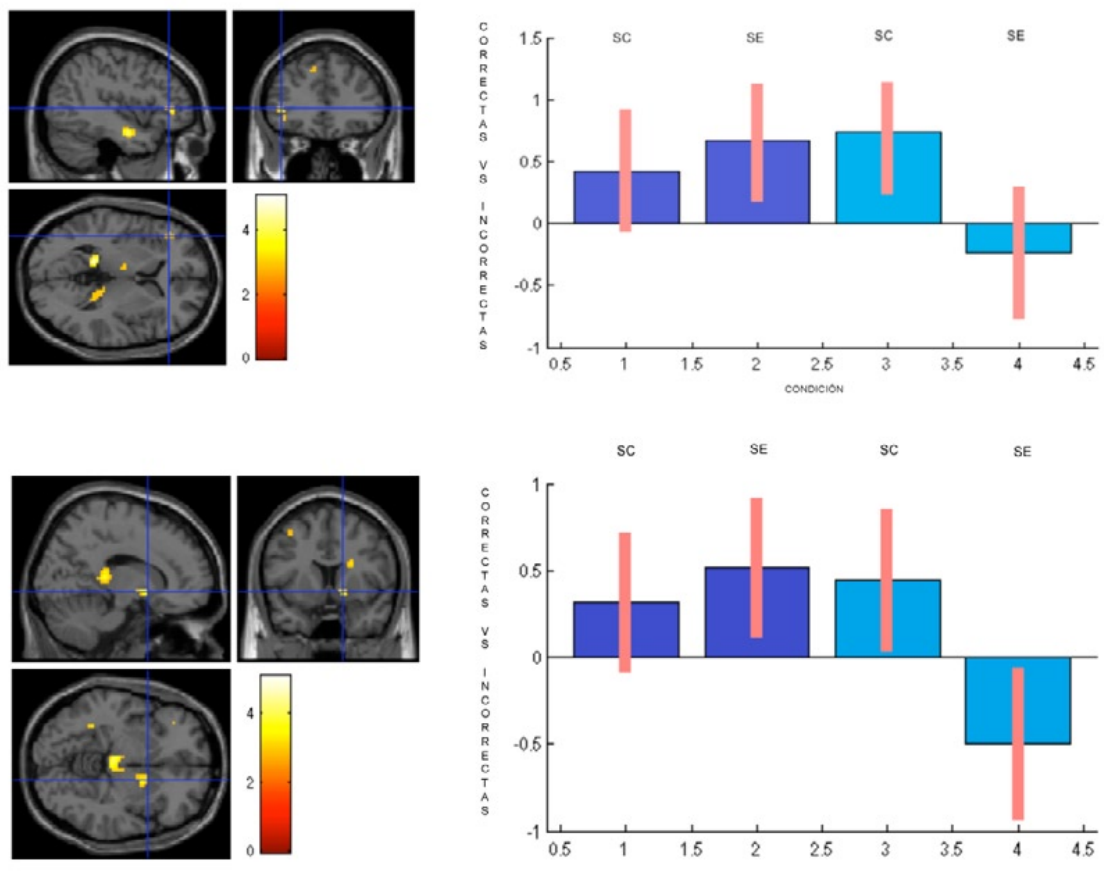

SC, Stop correcto; SE, Stop Erróneo. Azul oscuro, condición control: azul celeste, condición recompensa. La barra de colores representa los valores estadísticos del efecto de la interacción.

Figura 3. Efectos cerebrales en la interacción $(p<0,005$, a nivel de vóxel no corregido)

El análisis de correlación produjo una correlación positiva entre las puntuaciones en SR y la activación del GFI izq. obtenida en el efecto de interacción (véanse tabla 2 y figura 4).

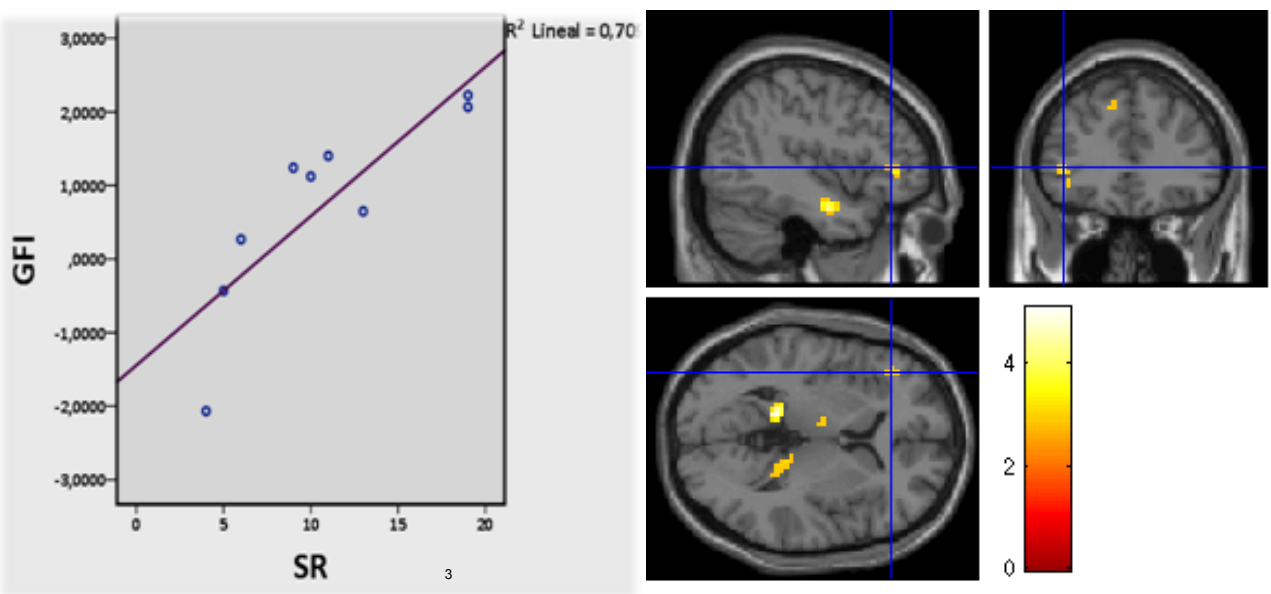

GFI, Giro Frontal Inferior; SR, Sensibilidad a la Recompensa. La barra de colores representa los valores estadísticos del efecto de la interacción.

Figura 4. Efectos de correlación entre el giro frontal inferior izquierdo y las puntuaciones en SR 


\section{Discusión y conclusiones}

El paradigma de señal de stop aplicado en este estudio ha mostrado las activaciones típicas en este tipo de procesos cognitivos. Así, nuestros resultados muestran los efectos cerebrales de la inhibición de la red fronto-parietal y en áreas estriatales en concordancia con la literatura previa (Casey y cols., 2002; Padmala y Pessoa, 2010; Aron y cols., 2007; Aron y Poldrack, 2006; Verbruggen y Logan, 2008). Por otra parte, cuando introducimos contingencias de refuerzo monetario en esta tarea, hemos observado que la actividad del GFI izquierdo y del estriado derecho pueden verse influenciadas por el hecho de recompensar o no la correcta ejecución en la tarea (Padmala y Pessoa, 2010; Padmala y Pessoa, 2011; Aarts y cols., 2011; Engelmann y cols., 2009; Locke y Braves, 2008). Por último, hemos mostrado que dicha actividad puede verse modulada por diferencias individuales en rasgos de personalidad relacionados con la motivación apetitiva como la SR (Ávila y cols., 2012; Ávila y Parcet, 2001).

Nuestros resultados muestran que durante procesos de inhibición conductual observamos la implicación del giro frontal inferior, giro frontal superior, córtex cingulado anterior y giro parietal. En este sentido, Verbruggen y Logan (2008) afirman que el giro frontal inferior se ha visto claramente implicado en procesos de inhibición de respuesta. Mientras que el córtex cingulado anterior está implicado en procesos de monitorización de la conducta. Por otra parte, el giro frontal superior se encargaría de procesar situaciones de conflicto (Padmala y Pessoa, 2011) y el giro parietal inferior de procesos de control atencional (Padmala y Pessoa, 2010). Por otra parte, obtuvimos una activación del estriado derecho, el cual se ha visto implicado en procesos de inhibición conductual y se ha relacionado con la saliencia del estímulo (Zink y cols., 2004). Estos autores definen un estímulo saliente como aquel que produce una activación y hacia el cual se dirigen las principales fuentes atencionales y conductuales. Puede que el contexto experimental utilizado en nuestro estudio haya hecho que el ER resulte incluso más saliente que la recompensa obtenida (Zink y cols., 2004).

Cuando analizamos la interacción entre los efectos de la tarea y el hecho de recompensar económicamente su correcta ejecución, se observó que tanto la actividad del GFI izq. como estriado derecho estaba influenciada por la motivación apetitiva. Estos resultados replican los obtenidos por Padmala y Pessoa (2010). En ambas áreas se observa un patrón de activación similar. Para la condición control observamos una mayor activación en los ensayos stop correctos (SC) que en los ensayos stop erróneos (SE). Sin embargo, para la condición recompensa ambas áreas se activan en los ensayos SC, pero se deactivan en los SE. Atendiendo a la implicación funcional de ambas áreas, podríamos especular que la deactivación del GFI izq. cuando no se consigue inhibir, podría producirse por la falta de procesamiento de la saliencia del ER por parte del estriado.

Las diferencias individuales en SR de los participantes correlacionaron de forma positiva con la actividad del GFI, lo cual está de acuerdo a lo que se obtuvo en un reciente estudio llevado a cabo por Ávila y cols. (2012), en el que correlacionaron las diferencias individuales en SR con la actividad de las áreas implicadas en una tarea de cambio de norma, en la cual se necesita de una importante implicación de procesos de control cognitivo para llevarla a cabo. Teniendo en cuenta que la SR es un rasgo de personalidad muy ligado a la actividad del sistema dopaminérgico y que este modula el flujo de información desde circuitos frontoestriatales ventrales, implicados en la recompensa y la motivación, hacia circuitos frontoestriatales dorsales, asociados con la cognición y la acción (Aarts, 2011), resulta conforme a lo esperado que la actividad del GFI se vea modulada por la actividad de los circuitos dopaminérgicos. De lo cual se infiere, que la actividad del GFI permite la inhibición de la respuesta y dicha actividad se ve incrementada cuando la inhibición conlleva la obtención de un refuerzo. Y a su vez, este 
aumento en la actividad del GFI podría ser más acusado si se tiene una puntuación alta en SR.

Respecto a la ejecución de este tipo de tareas cognitivas, no existe un consenso claro sobre cómo afecta el refuerzo monetario a variables conductuales de interés como por ejemplo el TR o el número de acierto de los ensayos stop (Padmala y Pessoa, 2011; Stoppel y cols, 2011; Padmala y Pessoa, 2010; Savine y Braver, 2010; Beck y cols., 2010; Pochon y cols., 2002; Rogers y cols., 2004). En este sentido, los resultados obtenidos en el presente estudio contradicen, en parte, a los resultados obtenidos en el único estudio previo similar al nuestro (Padmala y Pessoa, 2010). Es en la variable SSRT donde encontramos la contradicción más importante entre ambos estudios, ya que para Padmala y Pessoa (2010) el SSRT fue mayor en la condición recompensa que en la control, lo cual puede interpretarse como que a sus participantes les costó inhibir más en la condición recompensa que en la control, mientras que en el presente estudio sucede completamente lo contrario. Tales diferencias pueden ser debidas a la existencia de diferencias metodológicas entre ambos estudios; (1) Padmala y Pessoa (2010) no recompensaron los ensayos stop correctamente inhibidos tal y como hicimos nosotros. Esto puede conllevar a que los participantes elijan la estrategia de esperar la aparición de la señal de stop antes de responder al ER, ya que de este modo se aseguran la correcta ejecución en ambos tipos de ensayos. (2) Padmala y Pessoa (2010) utilizan un diseño de bloques según la condición, mientras que en el presente estudio se empleó un fast event-related. Por otra parte, respecto a la muestra utilizada, se debe destacar que es demasiado reducida $(n=9)$ para sacar conclusiones extrapolables a la población general y resta validez a los resultados obtenidos, y que no es equivalente en número respecto al sexo, por lo que se propone la ampliación de la muestra para su posible replicación o contrastación. A su vez, esta medida ofrecería una mayor variabilidad a los datos, y tal vez supliría la falta de correlación entre los datos conductuales y funcionales obtenidos. Por último, se destaca la elección del contraste stop correctos contra stop erróneos para entender las bases neuronales del control cognitivo durante una tarea de señal de «stop», ya que otros autores como Aron y Poldrack (2006) emplearon la estrategia de contrastar las imágenes stop correctas contra go correctas.

Con lo que se ha expuesto a lo largo del presente estudio, podemos sugerir que los efectos de la motivación apetitiva sobre la actividad de áreas cerebrales implicadas en procesos de control cognitivo puede ser modulada por rasgos de personalidad caracterizados por una alta sensibilidad a estímulos reforzadores como es la SR. En este sentido, tales implicaciones funcionales deberían tenerse en cuenta a la hora de evaluar y abordar enfermedades mentales que se han visto relacionadas con la falta de control cognitivo, la cual se manifiesta en problemas de desinhibición y impulsividad (Ávila y cols., 2008; Logan y cols., 1997), tales como, los trastornos alimentarios, adicciones, el trastorno por déficit de atención e hiperactividad (TDAH), el trastorno obsesivo-compulsivo (TOC) o incluso la manía (Aron y cols., 2007). Nuevos estudios con aproximaciones metodológicas más completas serían necesarios para ampliar y dar validez a los resultados obtenidos en este estudio.

\section{Referencias bibliográficas}

Aarts, E., Holstein, M. \& Cools, R. (2011). Striatal dopamine and teh interface between motivation and cognition. Frontiers inpsychology. 2, article 163.

Aron, A. \& Poldrack, R. (2006). Cortical and subcortical contributions to stop-signal response inhibition: role of the subthalamic nucleus. Journal of Neuroscience, 26 (9): 2424-2433.

Aron A., Durston, S., Eagle, D., Logan, G., Stinear, C. \& Stuphorn, V. (2007). Converging evidence for a fronto-basal-ganglia network for inhibitory control of action and cognition. The journal of neuroscience. 27(44), 11860-11864. 
Ávila, C., Garbin, G., Sanjuán, A., Forn, C., Barrós-Loscertales, A., Bustamante, J., RodríguezPujadas, A., Belloch, V. \& Parcet, M. (2012). Frontostrialt response to set switching is moderated by reward sensitivity. SCAN, 7, 423-430.

Ávila, C., Parcet, M. \& Barros, A. (2008). A cognitive neuroscience approach to individual differences in sensitivity to reward. Neurotoxicity Research. 14(2,3), 191-203.

Ávila, C. \& Parcet, M. (2001). Personality and inhibitory deficits in the stop-signal task: the mediating role of Gray's anxiety and impulsivity. Personality and individual differences. 31, 975-986.

Band, G., Van der Molen, M. \& Logan, G. D. (2003). Horse-race model simulations of the stopsignal procedure. Acta Psychologica. 112,105-142.

Beck, S., Locke, H., Savine, A., Jimura, K. \& Braver, T. (2010). Primary and Secondary Rewards Differentially Modulate Neural Activity Dynamics during Working Memory. Plos one. 5(2), e9252.

Casey, B. J., Thomas, K. M, Davidson, M. C., Kunz, K. \& Franzen, P. L. (2002). Dissociating striatal and hippocampal function developmentally with a stimulus-response. The Journal of Neuroscience, 22(9): 8647-8652.

Chambers, C.D., Garavan, H. \& Bellgrove, M. A. (2009). Insights into the neural basis of response inhibition from cognitive and clinical neuroscience. Neuroscience and Biobehavioral Reviews, 33:631-646.

Chevrier, A., Noseworthy, M. \& Schachar, R. (2007). Dissociation of Response Inhibition and Performance Monitoring in the Stop Signal Task Using Event-Related fMRI. Human Brain Mapping. 28,1347-1358.

Congdon, E., Mumford, J., Cohen, J., Galvan, A., Canli, T. \& Poldrack, R. (2012). Measurement and reliability of response inhibition. Frontiers in psychology, 3(37).

Duncan, J. \& Owen, A. (2000). Common regions of the human frontal lobe recruited by diverse cognitive demands. Trends Neuroscience. 23 (10), 475-483.

Engelmann, J., Damaraju, E., Padmala, S. \& Pessoa L. (2009). Combined effects of attention and motivation on visual task performance: transient and sustained motivational effects. Frontiers in human neuroscience. 3(4).

Friston, K. J., Holmes, A. P., Poline, J. B. y cols. (1995). Analysis of fMRI time-series revisited. Neuroimage, 2(1), 45-53.

Gilbert, A. \& Fiez, J. (2004). Integrating rewards and cognition in the frontal cortex. Cognitive and affective behavioural neuroscience. 4(4), 540-552.

Gray, J., Burgess, G., Schaefer, A., Yarkoni, T., Larsen, R. \& Braver, T. (2005). Affective personality differences in neural processing efficiency confirmed using fMRI. Cognitive, affective and behavioral neuroscience. 5(2),182-190.

Hahn, T., Dresler, T., Ehlis, A., Plichta, M., Heinzel, S., Polak, T., Lesch, K., Breuer, F., Jakob, P. \& Fallgatter, A. (2009) Neural response to reward anticipation is modulated by Gray's impulsivity. Neurolmage. 46, 1148-1153.

Johnstone, S., Dimoska, A., Smith, J., Barry, R., Pleffer, C., Chiswick, D. \& Clarke, A. (2007). The development of stop-signal and Go/Nogo response inhibition in children aged 7-12 years: Performance and event-related potential indices. International Journal of Psychophysiology. 63, 25-38.

Krebs, R., Schott, B., Schütze, H. \& Düzel, E. (2009). The novelty exploration bonus and its attentional modulation. Neuropsychologia. 47(11), 2272-2281.

Li, C., Krystal, J. \& Mathalon, D. (2005). Fore-period effect and stop-signal reaction time. Experimental Brain Research. 167, 305-309.

Li, C., Huang, C., Constable, R. \& Sinha, R. (2006). Imaging Response Inhibition in a Stop-Signal Task:Neural Correlates Independent of Signal Monitoring and Post-Response Processing. The Journal of Neuroscience. 26(1),186-192. 
Li, C., Huang, C., Yan, P., Bhagwagar, Z., Milivojevic, V. \& Sinha, R. (2008). Neural Correlates of Impulse Control During Stop Signal Inhibition in Cocaine-Dependent Men. Neuropsychopharmacology. 33, 1798-1806.

Locke, H. \& Braver, T. (2008). Motivational influences on cognitive control: behaviour, brain activation, and individual differences. Cognitive, affective and behavioural Neuroscience, 8(1), 99-112. Neuroimage. 62(3), 1841-1847.

Logan, G. D. \& Cowan, W. B. (1984). On the ability to inhibit thought and action: A theory of an act of control. Psychological Review, 91, 295-327.

Logan, G. D., Schachar, R. \& Tannock, R. (1997). Impulsivity and inhibitory control. Psychological Science. 8, 60-64.

O'Connor, D., Rossiter, S., Yücel, M., Lubman, D. \& Hester, R. (2012). Successful inhibitory control over an immediate reward is associated with attentional disengagement in visual processing areas. Neuroimage, 62 (3): 1841-1847.

Padmala, S. \& Pessoa, L. (2010). Interactions between cognition and motivation during response inhibition. Neuropsychologia, 48, 558-565.

Padmala, S. \& Pessoa, L. (2011). Reward reduces conflict by enhancing intentional control and biasing visual cortical processing. Journal of Cognitive Neuroscience. 23(11), 3419-3432.

Pessoa, L. \& Engelmann, J. (2010). Embedding reward signals into perception and cognition. Frontiers in neuroscience. 4:17.

Pochon, J., Levy, R., Fossati, P., Lehericy, S., Poline, J., Pillon, B., Le Bihan, D. \& Dubois, B. (2002). The neural system that bridges reward and cognition in humans: An fMRI study. Proceedings of the National Academy of Sciences of United States of America (PNAS). 99(8), 5669-5674.

Rogers, R., Ramnani, N., Mackay, C., Wilson, J., Jezzard, P., Carter, C. \& Smith, S. (2004). Distinct portions of anterior cingulate cortex and medial prefrontal cortex are activated by reward processing in separable phases of decision-making cognition. Biological Psychiatry. 55(6), 594-602.

Savine, A. \& Braver,T. (2010). Motivated cognitive control: Reward incentives modulate preparatory neural activity during task-switching. Journal of Neuroscience. 30(31), 10294-10305.

Simon, J., Walther, S., Fiebach, C., Friederich, H., Stippich, C., Weisbrod, M. \& Kaise, S. (2010). Neural reward processing is modulated by approach- and acoidance-related personality traits. Neuroimage. 49, 1868-1874.

Stoppel, A., Boehler, C., Strumpf, H., Heinze, H., Hopf, J. \& Schoenfeld, M. (2011). Neural processing of reward magnitude under varying attentional demands. Brain research. 1383, 218-229.

Tomporowski, P. \& Tinsley, V. (1996). Effects of memory demand and motivation on sustained attention in young and older aldults. The American journal of psychology. 109(2), 187-204.

Torrubia, R., Ávila, C., Moltó, J. \& Caseras, X. (2001). The Sensitivity to Punishment and Sensitivity to Reward Questionnaire (SPSRQ) as a measure of Gray's anxiety and impulsivity dimensions. Personality and individual differences, 31(6), 837-862. doi:10.1016/S01918869(00)00183-5.

Verbruggen, F. \& Logan, G. (2008). Response inhibition in the stop-signal paradigm. Trends in cognitive sciences. 12(11), 418-424.

Verbruggen, F., Logan, G. \& Stevens, M. (2008). STOP-IT: Windows executable software for the stop-signal paradigm. Behavior Research Methods. 40 (2), 479-483.

Zink, C., Pagnoni, G., Martin-Skurski, M., Chappelow, J. \& Berns, G. (2004), Human striatal responses to monetary reward depend on saliency. Neuron. 42, 509-517. 